\title{
Weighted Hardy-Type Inequalities in Variable Exponent Morrey-Type Spaces
}

\author{
Dag Lukkassen, ${ }^{1}$ Lars-Erik Persson, ${ }^{2,3}$ Stefan Samko, ${ }^{4}$ and Peter Wall ${ }^{2}$ \\ ${ }^{1}$ Narvik University College and Norut Narvik, P.O. Box 385, N-8505 Narvik, Norway \\ ${ }^{2}$ Department of Mathematics, Luleå University of Technology, SE 97187 Luleå, Sweden \\ ${ }^{3}$ Narvik University College, P.O. Box 385, N-8505, Narvik, Norway \\ ${ }^{4}$ Universidade do Algarve, FCT, Campus de Gambelas, Faro 8005-139, Portugal
}

Correspondence should be addressed to Lars-Erik Persson; lars-erik.persson@ltu.se

Received 20 April 2013; Accepted 3 September 2013

Academic Editor: Lars Diening

Copyright (C) 2013 Dag Lukkassen et al. This is an open access article distributed under the Creative Commons Attribution License, which permits unrestricted use, distribution, and reproduction in any medium, provided the original work is properly cited.

\begin{abstract}
We study the $p(\cdot) \rightarrow q(\cdot)$ boundedness of weighted multidimensional Hardy-type operators $H_{w}^{\alpha(\cdot)}$ and $\mathscr{H}_{w}^{\alpha(\cdot)}$ of variable order $\alpha(x)$, with radial weight $w(|x|)$, from a variable exponent locally generalized Morrey space $\mathscr{L}^{p(\cdot), \varphi(\cdot)}\left(\mathbb{R}^{n}, w\right)$ to another $\mathscr{L}^{q(\cdot), \psi(\cdot)}\left(\mathbb{R}^{n}, w\right)$. The exponents are assumed to satisfy the decay condition at the origin and infinity. We construct certain functions, defined by $p, \alpha$, and $\varphi$, the belongness of which to the resulting space $\mathscr{L}^{q(\cdot), \psi(\cdot)}\left(\mathbb{R}^{n}, w\right)$ is sufficient for such a boundedness. Under additional assumptions on $\varphi / w$, this condition is also necessary. We also give the boundedness conditions in terms of Zygmund-type integral inequalities for the functions $\varphi$ and $\varphi / w$.
\end{abstract}

\section{Introduction}

Influenced by various applications, for instance, mechanics of the continuum medium and variational problems, in the last two decades the study of various mathematical problems in the spaces with nonstandard growth attracts the attention of researchers in various fields. This notion relates first of all to the generalized Lebesgue spaces $L^{p(\cdot)}(\Omega), \Omega \subseteq \mathbb{R}^{n}$, known also as Lebesgue spaces with variable exponent $p(x)$. We refer to the existing books [1-3] in the field.

This variable exponent boom naturally touched Morrey spaces. Morrey spaces $\mathscr{L}^{p, \lambda}$ (with constant exponents) in its classical version were introduced in [4] in relation to the study of partial differential equations and presented in various books; see, for example, [5-7]; we refer also to a recent overview of Morrey spaces in [8], where various generalizations of Morrey spaces may be also found.

They were widely investigated during the last decades, including the study of classical operators of harmonic analysis, maximal, singular, and potential operators on Morrey spaces, and their generalizations were studied. We refer for instance to papers [9-16] and the references therein; in particular, Hardy operators in Morrey type spaces with constant $p$ were studied in [17-20].

The Morrey spaces $L^{p(\cdot), \lambda(\cdot)}(\Omega)$ with variable exponents $\lambda(\cdot)$ and $p(\cdot)$ were introduced and studied in [21-23]. Generalized Morrey spaces $\mathscr{M}^{p(\cdot), \omega(\cdot)}(\Omega), \Omega \subseteq \mathbb{R}^{n}$ with variable exponents were studied in [24]; see also another version of Morrey-type spaces in [25]; we also refer to [26] for the socalled complementary Morrey spaces of variable order in the spirit of ideas of [24].

In the above cited paper maximal, singular, and potential operators were studied. This paper seems to be the first one where Hardy-type integral inequalities are studied in Morreytype spaces with variable exponents. Concerning Hardy-type inequalities and related problems and applications, we refer to the books $[27,28]$.

The paper is organized as follows. In Section 2, we give necessary preliminaries on variable exponent Lebesgue spaces. In Section 3, we define our main object-variable exponent Morrey spaces and prove important weighted estimates of functions in Morrey spaces; see Theorem 10.

By means of these estimates in Section 4, we prove our main statements for Hardy operators in variable exponent 
generalized Morrey spaces. We also consider the necessity of the obtained conditions. In Section 4.4, under some additional assumptions on $\varphi(0, r)$ we obtain the boundedness conditions in a different form via Zygmund-type conditions on $\varphi(0, r) / w(r)$ and provide a direct relation between $\varphi(0, r)$ and $\psi(0, r)$.

In Theorem 18 of this section, we specially single out the nonweighted case where we show that the Hardy inequalities in Morrey spaces are completely determined by the values $p(0)$ and $p(\infty)$.

In the appendix we recall some notions related to the Bary-Zygmund-Stechkin class and Matuszewska-Orlicz indices which sporadically are used in the paper.

\section{Preliminaries on Variable Exponent Lebesgue Spaces}

We first recall the basic definitions related to variable exponent spaces. By $\Omega$, we always denote an open set in $\mathbb{R}^{n}, \Omega \subseteq$ $\mathbb{R}^{n}$, and $\ell=\operatorname{diam} \Omega, 0<\ell \leq \infty$. Let also $B(x, r)=\left\{y \in \mathbb{R}^{n}\right.$ : $|x-y|<r\}$ and $\widetilde{B}(x, r)=B(x, r) \cap \Omega$.

Let $p(\cdot)$ be a measurable function on $\Omega$ with values in $[1, \infty)$. We suppose that

$$
1 \leq p_{-} \leq p(x) \leq p_{+}<\infty,
$$

where $p_{-}:=\inf _{x \in \Omega} p(x)>1, p_{+}:=\sup _{x \in \Omega} p(x)<\infty$.

We denote by $L^{p(\cdot)}(\Omega)$ the space of all measurable functions $f(x)$ on $\Omega$ such that $I_{p(\cdot)}(f)=\int_{\Omega}|f(x)|^{p(x)} d x<\infty$. Equipped with the norm $\|f\|_{p(\cdot)}=\inf \left\{\eta>0: I_{p(\cdot)}(f / \eta) \leq 1\right\}$, this is a Banach function space. For the basics on variable exponent Lebesgue spaces, we refer to $[2,29,30]$.

We denote by $p^{\prime}(\cdot)=p(x) /(p(x)-1), x \in \Omega$, the conjugate exponent of $p(x)$. The notation $\mathbb{P}^{\log }(\Omega)$ will stand for the set of variable exponents $p(\cdot)$ satisfying condition (1) and the local log-condition

$$
|p(x)-p(y)| \leq \frac{A}{-\ln |x-y|}, \quad|x-y| \leq \frac{1}{2} x, y \in \Omega,
$$

where $A=A(p)>0$ does not depend on $x, y$.

We will use also the following decay conditions:

$$
\begin{array}{cl}
|p(x)-p(0)| \leq \frac{A_{0}}{|\ln | x||}, & |x| \leq \frac{1}{2}, \\
\left|p(x)-p_{\infty}\right| \leq \frac{A_{\infty}}{|\ln | x||}, & |x| \geq 2 .
\end{array}
$$

For brevity, by $\mathscr{P}_{0, \infty}(\Omega)$ we denote the set of bounded measurable functions (not necessarily with values in $[1, \infty)$ ), which satisfy the decay conditions (3) and (4).

Let $p$ satisfy the log-condition (2). The inequality

$$
\left\|\chi_{B(x, r)}\right\|_{L^{p(\cdot)}(\Omega)} \leq C r^{n / p(x)}
$$

for bounded open sets $\Omega$ is known and proved in [31]. For unbounded sets, if, besides (2), the exponent $p$ satisfies the decay condition (3), then we have

$$
\left\|\chi_{B(x, r)}\right\|_{L^{p(\cdot)}(\Omega)} \leq C r^{n / p_{r}(x)}
$$

where

$$
p_{r}(x):= \begin{cases}p(x), & \text { if } 0<r<1, \\ p(\infty), & \text { if } r \geq 1 .\end{cases}
$$

See [2, Corollary 4.5.9]. The following lemma was proved in [32], Lemma 2, and a simpler proof given in [33].

Lemma 1. Let $p(x)$ be a bounded function. Then

$$
\begin{array}{ll}
\frac{1}{c_{0}} r^{n / p(0)} \leq\left\|\chi_{B(0, D r) \backslash B(0, r)}\right\|_{p(\cdot)} \leq c_{0} r^{n / p(0)} & \text { for } 0<r \leq a<\infty, \\
\frac{1}{c_{\infty}} r^{n / p_{\infty}} \leq\left\|\chi_{B(0, D r) \backslash B(0, r)}\right\|_{p(\cdot)} \leq c_{\infty} r^{n / p_{\infty}} & \text { for } 0<b \leq r<\infty,
\end{array}
$$

under conditions (3) or (4), respectively, where $D>1$ and $c_{0} \geq$ 1 and $c_{\infty} \geq 1$ depend on $D$ and $a, b$, but do not depend on $r$.

We will use a consequence of the estimates of Lemma 1 in the form

$$
\left\|\chi_{B(0,2 r) \backslash B(0, r)}\right\|_{p(\cdot)} \leq c_{0} r^{n / p_{*}(r)}, \quad r \in \mathbb{R}_{+},
$$

where we denoted

$$
p_{*}(r)= \begin{cases}p(0), & r \leq 1 \\ p(\infty), & r \geq 1\end{cases}
$$

for brevity.

We refer to the appendix for the definition of the classes $\bar{W}\left(\mathbb{R}_{+}\right)$and $\underline{W}\left(\mathbb{R}_{+}\right)$used in the following lemma.

Lemma 2. Let $p \in \mathscr{P}_{0, \infty}\left(\mathbb{R}^{n}\right)$ and $1 \leq p_{-} \leq p_{+}<\infty$ and $a$ function $a(x, r)$ belongs to $\bar{W}\left(\mathbb{R}_{+}\right) \cap \underline{W}\left(\mathbb{R}_{+}\right)$with respect to $r$ uniformly in $x$. Then

$$
\left\|a(x, r) \chi_{B(0, r)}\right\|_{p(\cdot)} \leq C \int_{0}^{r} t^{n / p_{*}(t)} a(x, t) \frac{d t}{t}, \quad r>0,
$$

where $C>0$ does not depend on $r$ and $x$.

Proof. Let $B_{k(0, r)}:=B\left(0,2^{-k} r\right) \backslash B\left(0,2^{-k-1} r\right)$. We have

$$
\left\|a(x, r) \chi_{B(0, r)}\right\|_{p(\cdot)} \leq \sum_{k=0}^{\infty}\left\|a(x, r) \chi_{B_{k}(0, r)}\right\|_{p(\cdot)} .
$$

Since $a \in \bar{W}$, we obtain

$$
\left\|a(x, r) \chi_{B(0, r)}\right\|_{p(\cdot)} \leq C \sum_{k=0}^{\infty} a\left(x, 2^{-k} r\right)\left\|\chi_{B\left(x, 2^{-k} r\right)}\right\|_{p(\cdot)} .
$$

By (9), we have

$$
\left\|\chi_{B\left(0,2^{-k} r\right)}\right\|_{p(\cdot)} \leq c\left(2^{-k} r\right)^{n / p_{*}\left(2^{-k} r\right)} .
$$

Therefore,

$$
\begin{aligned}
\left\|a(x, r) \chi_{B(0, r)}\right\|_{p(\cdot)} & \leq C \sum_{k=0}^{\infty} a\left(x, 2^{-k} r\right)\left(2^{-k} r\right)^{n / p_{*}\left(2^{-k} r\right)} \\
& \leq \frac{C}{\ln 2} \int_{0}^{r} t^{n / p_{*}(t)} a(x, t) \frac{d t}{t}
\end{aligned}
$$


and we arrive at (11). The last passage to the integral is verified in the standard way with the use of the monotonicity properties of the function $t^{n / p_{*}(t)} a(x, t)$ in $t$, imposed by the assumptions of the lemma on $a(x, t)$ as

$$
\begin{aligned}
& \int_{0}^{r} t^{n / p_{*}(t)} a(x, t) \frac{d t}{t} \\
& \quad=\sum_{k=0}^{\infty} \int_{2^{-k-1} r}^{2^{-k} r} t^{n / p_{*}(t)} a(x, t) \frac{d t}{t} \\
& \quad \geq \sum_{k=0}^{\infty} a\left(x, 2^{-k} r\right)\left(2^{-k} r\right)^{+n / p_{*}\left(2^{-k} r\right)} \int_{2^{-k-1} r}^{2^{-k} r} \frac{d t}{t} \\
& \quad=\ln 2 \sum_{k=0}^{\infty} a\left(x, 2^{-k} r\right)\left(2^{-k} r\right)^{n / p_{*}\left(2^{-k} r\right)} .
\end{aligned}
$$

The class $\mathbb{Z}^{\beta_{0}, \beta_{\infty}}\left(\mathbb{R}_{+}^{1}\right)$ used in the following corollary is defined in (A.18).

Corollary 3. Let $a(x, \cdot)$ belong to the class $\mathbb{Z}^{\beta_{0}, \beta_{\infty}}\left(\mathbb{R}_{+}^{1}\right)$ uniformly in $x$, where $\beta_{0}=n / p(0)$ and $\beta_{\infty}=n / p(\infty)$. Then

$$
\left\|a(x, r) \chi_{B(0, r)}\right\|_{p(\cdot)} \leq C r^{n / p_{*}(r)} a(x, r), \quad r>0 .
$$

Proof. The statement follows from (11) by the definition of the class $\mathbb{Z}^{\beta_{0}, \beta_{\infty}}\left(\mathbb{R}_{+}^{1}\right)$.

Corollary 4. Let $p \in \mathscr{P}_{0, \infty}\left(\mathbb{R}^{n}\right)$ and $1 \leq p_{-} \leq p_{+}<\infty$ and $a$ bounded function $\nu(x)$ satisfies the conditions

$$
\inf _{x \in \mathbb{R}^{n}}[n+v(x) p(0)], \quad \inf _{x \in \mathbb{R}^{n}}[n+v(x) p(\infty)]>0 .
$$

Then

$$
\left\||y|^{\nu(x)} \chi_{B(0, r)}\right\|_{p(\cdot)} \leq c r^{\nu(x)+\left(n / p_{*}(r)\right)}, \quad r>0,
$$

where $c>0$ does not depend on $r$ and $x$.

Proof. The statement follows directly from (11) with $a(x, r)=$ $r^{\nu(x)}$, since $\int_{0}^{r} t^{\nu(x)+\left(n / p_{*}(t)\right)}(d t / t)=r^{\nu(x)+(n / p(0))} /(\nu(x)+$ $(n / p(0)))$ when $0<r \leq 1$ and $\int_{0}^{r} t^{\nu(x)+\left(n / p_{*}(t)\right)}(d t / t)=$ $1 /(\nu(x)+(n / p(0)))+\left(r^{\nu(x)+(n / p(\infty))}-1\right) /(\nu(x)+(n / p(\infty)))$ when $r \geq 1$.

\section{Variable Exponent Morrey Spaces}

3.1. Definitions and Some Auxiliary Results for Variable Exponent Morrey Spaces. Let $\varphi(r)$ be a nonnegative function on $[0, \ell]$, positive on $(0, \ell]$. Morrey type spaces, called also generalized Morrey spaces, with constant $p, 1 \leq p<\infty$ are known in two versions: global $\mathscr{L}^{p, \varphi}(\Omega)$ and local $\mathscr{L}_{\text {loc; } x_{0}}^{p, \varphi}(\Omega)$ (we refer, for instance, to the survey paper [8]) and are defined as the spaces of functions $f \in L_{\text {loc }}^{p}(\Omega)$ such that

$$
\begin{gathered}
\sup _{x \in \Omega, r>0} \frac{\|f\|_{L^{p}(\widetilde{B}(x, r))}}{\varphi(r)}<\infty, \\
\sup _{r>0} \frac{\|f\|_{L^{p}\left(\widetilde{B}\left(x_{0}, r\right)\right)}}{\varphi(r)}<\infty,
\end{gathered}
$$

respectively, where $x_{0} \in \Omega$.

Morrey spaces with variable exponent $p(x)$ corresponding to the classical case $\varphi(r)=r^{\lambda / p}$, but with variable $\lambda(x)$ as well, were introduced and studied in [21]. More general approach admitting the variable function $\varphi(x, r)$ were studied in $[24,25]$.

Following [24], we introduce the variable exponent Morrey-type space by the definition below, but note that our notation differs from that of [24].

Definition 5. Let $1 \leq p_{-} \leq p_{+}<\infty$ and let $\varphi(x, t)$ be a nonnegative function almost increasing in $r$ uniformly in $x \in \Omega$. The generalized variable exponent Morrey space $\mathscr{L}^{p(\cdot), \varphi(\cdot)}(\Omega)$ is defined by the norm

$$
\|f\|_{\mathscr{L}^{p(\cdot), \varphi(\cdot)}}=\sup _{x \in \Omega, r>0} \frac{1}{\varphi(x, r)}\|f\|_{L^{p(\cdot)}(\widetilde{B}(x, r))} .
$$

We will also refer to the space $\mathscr{L}^{p(\cdot), \varphi(\cdot)}(\Omega)$ as global generalized variable exponent Morrey space in contrast to its local version $\mathscr{L}_{x_{0} ; \text { loc }}^{p(\cdot), \varphi(\cdot)}(\Omega)$ defined by the norm

$$
\|f\|_{\mathscr{L}_{x_{0} ; \mathrm{loc}}^{p(\cdot) \varphi}}=\sup _{r>0} \frac{1}{\varphi\left(x_{0}, r\right)}\|f\|_{L^{p(\cdot)}\left(\widetilde{B}\left(x_{0}, r\right)\right)},
$$

where $x_{0} \in \Omega$.

For $w$ a weight function on $\Omega$, the weighted Morrey space $\mathscr{L}^{p, \varphi}(\Omega, w)$ is defined by $L^{p, \varphi}(\Omega, w):=\left\{f: w f \in L^{p, \varphi}(\Omega)\right\}$.

By the definition of the norm in the variable exponent Lebesgue space, we the can also write that

$$
\begin{aligned}
& \|f\|_{\mathscr{L}^{p(\cdot), \varphi(\cdot)}} \\
& \quad=\sup _{x \in \Omega, r>0} \inf \left\{\lambda=\lambda(x, r)>0: \int_{\widetilde{B}(x, r)}\left|\frac{f(y)}{\lambda \varphi(x, r)}\right|^{p(y)} d y \leq 1\right\} .
\end{aligned}
$$

From which one can see that for bounded exponents $p$ one has

$$
f \in \mathscr{L}^{p(\cdot), \varphi(\cdot)}(\Omega) \Longleftrightarrow \sup _{x, r} \int_{\widetilde{B}(x, r)}\left|\frac{f(y)}{\varphi(x, r)}\right|^{p(y)} d y<\infty .
$$

The following lemma provides some minimal assumptions on the function $\varphi(x, r)$ under which the so-defined spaces contain "nice" functions. 
Lemma 6. Let $\leq p_{-} \leq p_{+}<\infty$. Under the decay condition

$$
\left|p(x)-p\left(x_{0}\right)\right| \leq \frac{A_{0}}{|\ln | x-x_{0}||}, \quad\left|x-x_{0}\right| \leq \frac{1}{2},
$$

the assumption

$$
\sup _{r>0} \frac{[\min \{1, r\}]^{n / p\left(x_{0}\right)}}{\varphi\left(x_{0}, r\right)}<\infty
$$

is sufficient for bounded functions $f$ with compact support (in the case of unbounded set $\Omega$ ) to belong to the local Morrey space $\mathscr{L}_{x_{0} ; l o c}^{p(\cdot), \varphi(\cdot)}(\Omega)$. Similarly under the log-condition (2), the condition

$$
\sup _{x \in \Omega, r>0} \frac{[\min \{1, r\}]^{n / p(x)}}{\varphi(x, r)}<\infty
$$

guarantees that such functions belong to the global Morrey space $\mathscr{L}^{p(\cdot), \varphi(\cdot)}(\Omega)$.

Proof. Use (19) and (5), respectively.

Everywhere in the sequel we assume that the assumptions (26) and (27) for the spaces $\mathscr{L}_{x_{0} ; \text { loc }}^{p(\cdot) \varphi(\cdot)}(\Omega)$ and $\mathscr{L}^{p(\cdot), \varphi(\cdot)}(\Omega)$, respectively, are satisfied.

We need the following lemma on variable exponent powers of functions in Bary-Stechkin class. For this class, Matuszewska-Orlicz indices, and all the related notation, we refer to the appendix.

Lemma 7. Let $1 \leq p_{-} \leq p_{+}<\infty, \varphi \in \bar{W}([0, \ell]) \cap \underline{W}([0, \ell])$, and $p$ satisfy the decay condition (3). Then

$$
\frac{1}{C} \varphi^{p(0)}(r) \leq \varphi^{p(y)}(r) \leq C \varphi^{p(0)}(r), \quad \text { for }|y| \leq r \leq \ell,
$$

where $\ell<\infty$ and $C \geq 1$ do not depend on $r$ and $y$.

Proof. We have to prove that $1 / C \leq[\varphi(r)]^{p(y)-p(0)} \leq C$; that is,

$$
|p(y)-p(0)| \cdot|\ln \varphi(r)| \leq C_{1}(=\ln C) .
$$

It suffices to consider the case $r \leq 1$. By Theorem A.4, the assumption $\varphi \in \bar{W}([0, \ell]) \cap \underline{W}([0, \ell])$ implies that the function $\varphi$ has finite indices $m(\varphi)$ and $M(\varphi)$, and (A.11) holds. Bounds in (A.11) yield the inequality $|\ln \varphi(r)| \leq c_{1}+c_{2}|\ln r|$ with some positive $c_{1}$ and $c_{2}$. Then (29) follows from the decay condition at the origin, since $\ln (1 / r) \leq \ln (1 /|y|)$.

In papers $[17,18]$, there were given various conditions for radial type functions to belong to Morrey spaces with nonvariable characteristics. The reader can easily adjust them for the case when they are variable. We do not dwell on this, but in the next lemma we give a certain example of a function in the space $\mathscr{L}_{0 ; l o c}^{p(\cdot),(\cdot)}\left(\mathbb{R}^{n}\right)$, important for our further goals.
Lemma 8. Let $\leq p_{-} \leq p_{+}<\infty$ and $p$ satisfy the decay condition (3) at the origin and $\varphi(0, \cdot) \in \bar{W}\left(\left[0, \varepsilon_{0}\right]\right) \cap \underline{W}\left(\left[0, \varepsilon_{0}\right]\right)$ for some $\varepsilon_{0}>0$, and

$$
\int_{0}^{h} \frac{\varphi(0, t)}{t} d t \leq c \varphi(0, h)
$$

for small $h$ and some $c>0$. Then the function $f_{0}(x)=$ $\left(\varphi(0,|x|) /|x|^{n / p(x)}\right) \chi_{B(0, R)}(x)$, where $0<R<\infty$, belongs to $\mathscr{L}_{0 ; l o c}^{p(\cdot), \varphi(\cdot)}\left(\mathbb{R}^{n}\right)$.

If additionally we suppose that $p$ satisfies the decay condition (4) at infinity, $\varphi(0, \cdot) \in \bar{W}([N, \infty)) \cap \underline{W}([N, \infty))$ for some $N>0$, the inequality (30) holds also for large $h, \varphi(0, r) \geq c>0$ for large $r \rightarrow \infty$ and $p_{-}=p(\infty)$; then the same holds with $R=\infty$.

Proof. We have to check that

$$
\begin{aligned}
& \sup _{r} \int_{B(0, r)}\left|\frac{f_{0}(y)}{\varphi(0, r)}\right|^{p(y)} d y \\
& \quad=\sup _{0<r<R} \int_{B(0, r)}\left|\frac{\varphi(0,|y|)}{\varphi(0, r)}\right|^{p(y)} \frac{d y}{|y|^{n}}<\infty .
\end{aligned}
$$

By Lemma 7, this is guaranteed by the condition

$$
\sup _{r} \frac{1}{\varphi^{p(0)}(0, r)} \int_{0}^{r} \varphi^{p(0)}(0, t) \frac{d t}{t}<\infty
$$

The latter is equivalent to the condition $m\left(\varphi^{p(0)}(0, t)\right)>0$, that is, $m(\varphi(0, t))>0$, which in its turn is equivalent to (30) and consequently holds.

In the case of $R=\infty$, the proof follows the same lines. This time instead of (31) it suffices only to check that

$$
\sup _{r>N} \int_{B(0, r)}\left|\frac{\varphi(0,|y|)}{\varphi(0, r)}\right|^{p(y)} \frac{d y}{|y|^{n}}<\infty
$$

for some large $N>0$. Here $\varphi(0,|y|)^{p(y)} \sim \varphi(0,|y|)^{p(\infty)}$ by the decay condition at infinity imposed on $p$. Therefore,

$$
\sup _{r>N} \int_{B(0, r)}\left|\frac{\varphi(0,|y|)}{\varphi(0, r)}\right|^{p(y)} \frac{d y}{|y|^{n}} \leq \sup _{r>N} \int_{B(0, r)}\left|\frac{\varphi(0,|y|)}{\varphi(0, r)}\right|^{p(\infty)} \frac{d y}{|y|^{n}}
$$

after which the arguments are similar to those for the case $R<\infty$.

Corollary 9. Let $1 \leq p_{-} \leq p_{+}<\infty$ and $p$ satisfy the decay conditions (3) and (4), $\varphi(0, \cdot) \in \bar{W}\left(\mathbb{R}_{+}\right) \cap \underline{W}\left(\mathbb{R}_{+}\right)$, and the inequality (30) holds for all $h \in \mathbb{R}_{+}, \varphi(0, r) \geq c>0$ for large $r \rightarrow \infty$ and $p_{-}=p(\infty)$; then

$$
\frac{\varphi(0,|x|)}{|x|^{n / p_{*}(|x|)}} \in \mathscr{L}_{0 ; l o c}^{p(\cdot), \varphi(\cdot)}\left(\mathbb{R}^{n}\right) .
$$




\subsection{Some Weighted Estimates of Functions in Morrey Spaces}

Theorem 10. Let $1 \leq p_{-} \leq p_{+}<\infty$ and $p \in \mathscr{P}_{0, \infty}\left(\mathbb{R}^{n}\right)$. Suppose also that $u \in \underline{W}\left(\mathbb{R}_{+}\right), \varphi(0, \cdot) / u(\cdot) \in \underline{W}\left(\mathbb{R}_{+}\right)$and $v \in$ $\bar{W}\left(\mathbb{R}_{+}^{1}\right), v(\cdot) \varphi(0, \cdot) \in \bar{W}\left(\mathbb{R}_{+}^{1}\right)$. Then

$$
\begin{aligned}
& \int_{|z|<r} \frac{|f(z)|}{u(|z|)} d z \leq c \mathscr{A}(r)\|f\|_{\mathcal{L}_{0 ;:>c c}^{p(\cdot),(\cdot)},}, \\
& \int_{|z|>r} v(|z|)|f(z)| d z \leq c \mathscr{B}(r)\|f\|_{\mathscr{L}_{0 ; 0 c c}^{p(\cdot), \varphi(\cdot)}}
\end{aligned}
$$

with

$$
\begin{aligned}
& \mathscr{A}(r)=\int_{0}^{r} t^{\left(n / p_{*}(t)\right)-1} \frac{\varphi(0, t)}{u(t)} d t, \\
& \mathscr{B}(r)=\int_{r}^{\infty} t^{\left(n / p_{*}^{\prime}(t)\right)-1} \varphi(0, t) v(t) d t,
\end{aligned}
$$

where $r \in \mathbb{R}_{+}$and $c>0$ do not depend on $r$ and $f$.

Proof. We have

$$
\int_{|z|<r} \frac{|f(z)|}{u(|z|)} d z=\sum_{k=0}^{\infty} \int_{B_{k}(y)} \frac{|f(z)|}{u(|z|)} d z
$$

where $B_{k}(y)=\left\{z: 2^{-k-1} r<|z|<2^{-k} r\right\}$. Making use of the fact that there exists a $\beta$ such that $t^{\beta} u(t)$ is almost decreasing, we observe that $1 / u(|z|) \leq C / u\left(2^{-k} r\right)$ on $B_{k}(y)$. Applying this in (38) and making use of the Hölder inequality with the exponent $p(x)$, we obtain

$$
\begin{aligned}
& \int_{|z|<r} \frac{|f(z)|}{u(|z|)} d z \\
& \leq \sum_{k=0}^{\infty} \frac{\left\|\chi_{B_{k}}\right\|_{L^{p^{\prime}(\cdot)\left(B_{k}\right)}}}{u\left(2^{-k} r\right)} \cdot\|f\|_{L^{p(\cdot)}\left(B\left(0,2^{-k} r\right)\right)} .
\end{aligned}
$$

By (9), we have $\left\|\chi_{B_{k}}\right\|_{L^{p^{\prime}(\cdot)\left(B_{k}\right)}} \leq C\left(2^{-k} r\right)^{n / p_{*}\left(2^{-k} r\right)}$, so that

$$
\begin{aligned}
& \int_{|z|<r} \frac{|f(z)|}{u(|z|)} d z \\
& \quad \leq C \sum_{k=0}^{\infty} \frac{\left(2^{-k} r\right)^{n / p_{*}^{\prime}\left(2^{-k} r\right)} \varphi\left(0,2^{-k} r\right)}{u\left(2^{-k} r\right)}\|f\|_{\mathcal{L}_{0 ; j \mathrm{loc}}^{\text {p(., })(\cdot)}}
\end{aligned}
$$

It remains to prove that

$$
\sum_{k=0}^{\infty} \frac{\left(2^{-k} r\right)^{n / p_{*}^{\prime}\left(2^{-k} r\right)} \varphi\left(0,2^{-k} r\right)}{u\left(2^{-k} r\right)} \leq C \mathscr{A}(r) .
$$

We have $\mathscr{A}(r)=\sum_{k=0}^{\infty} \int_{2^{-k-1} r}^{2^{-k}} t^{\left(n / p_{*}^{\prime}(t)\right)-1}(\varphi(t) / u(t)) d t$. Since the function $t^{n / p_{*}^{\prime}(t)}$ is increasing for all $t \in \mathbb{R}_{+}$and the function $\varphi(0, t) / t^{b} u(t)$ is almost decreasing with some $b$, we obtain

$$
\mathscr{A}(r) \geq C \sum_{k=0}^{\infty}\left(2^{-k} r\right)^{n / p_{*}^{\prime}\left(2^{-k} r\right)} \frac{\varphi\left(0,2^{-k} r\right)}{u\left(2^{-k} r\right)} \geq C,
$$

which proves (41) and completes the proof of the first inequality in (36).

For the second inequality in (36), we proceed in a similar way as

$$
\int_{|z|>r} v(t)|f(z)| d z=\sum_{k=0}^{\infty} \int_{B^{k}(y)} v(z)|f(z)| d z,
$$

where $B^{k}(y)=\left\{z: 2^{k} r<|z|<2^{k+1} r\right\}$. Since there exists a $\beta \in \mathbb{R}$ such that $t^{\beta} v(t)$ is almost increasing, we obtain

$$
\begin{aligned}
& \sum_{k=0}^{\infty} \int_{B^{k}(y)} v(|z|)|f(z)| d z \\
& \quad \leq C \sum_{k=0}^{\infty} v\left(2^{k+1} r\right) \int_{B^{k}(y)}|f(z)| d z .
\end{aligned}
$$

Applying the Hölder inequality with the variable exponent $p(\cdot)$ and taking (9) into account, we get

$$
\begin{aligned}
& \int_{|z|>r} v(|z|)|f(z)| d z \\
& \leq C \sum_{k=0}^{\infty} v\left(2^{k+1} r\right)\left\|\chi_{B_{k}(y)}\right\|_{p^{\prime}(\cdot)}\left\|f \chi_{B\left(0,2^{k+1} r\right)}\right\|_{p(\cdot)} \\
& \leq C \sum_{k=1}^{\infty} v\left(2^{k} r\right)\left(2^{k} r\right)^{n / p_{*}^{\prime}\left(2^{k} r\right)} \varphi\left(0,2^{k} r\right) \cdot\|f\|_{\mathcal{L}_{0 ; l o c}^{p(j),(\cdot)}} .
\end{aligned}
$$

It remains to prove that $\sum_{k=1}^{\infty} v\left(2^{k} r\right)\left(2^{k} r\right)^{n / p_{*}^{\prime}\left(2^{k} r\right)} \varphi\left(2^{k} r\right) \leq$ $C \mathscr{B}(r)$, which easily follows by the monotonicity of the involved functions as

$$
\begin{aligned}
\mathscr{B}(r) & =\sum_{k=0}^{\infty} \int_{2^{k} r}^{2^{k+1} r} t^{n /\left(p_{*}^{\prime}(t)-1\right)} v(t) \varphi(t) d t \\
& \geq C \sum_{k=0}^{\infty} v\left(2^{k} r\right) \varphi\left(2^{k} r\right)\left(2^{k} r\right)^{n / p_{*}^{\prime}\left(2^{k} r\right)} .
\end{aligned}
$$

\section{On Weighted Hardy Operators in Generalized Morrey Spaces}

4.1. Pointwise Estimations. We consider the following generalized Hardy operators:

$$
\begin{gathered}
H_{w}^{\alpha(\cdot)} f(x)=|x|^{\alpha(x)-n} w(|x|) \int_{|y|<|x|} \frac{f(y) d y}{w(|y|)}, \\
\mathscr{H}_{w}^{\alpha(\cdot)} f(x)=|x|^{\alpha(x)} w(|x|) \int_{|y|>|x|}, \frac{f(y) d y}{|y|^{n} w(|y|)},
\end{gathered}
$$

where $\alpha(x)$ is a non-negative measurable function on $\mathbb{R}^{n}$. In the one-dimensional case, their versions

$$
\begin{aligned}
& H_{w}^{\alpha(\cdot)} f(x)=x^{\alpha(x)-1} w(x) \int_{0}^{x} \frac{f(t) d t}{w(t)}, \\
& \mathscr{H}_{w}^{\alpha(\cdot)} f(x)=x^{\alpha(x)} w(x) \int_{x}^{\infty} \frac{f(t) d t}{t w(t)}, \quad x>0
\end{aligned}
$$


on the half-axis $\mathbb{R}_{+}^{1}$ may be also admitted, so that the sequel $\mathbb{R}^{n}$ with $n=1$ may be read either as $\mathbb{R}^{1}$ or $\mathbb{R}_{+}^{1}$.

We also use the notation $H^{\alpha(\cdot)}=\left.H_{w}^{\alpha(\cdot)}\right|_{w \equiv 1}$.

Our next result on the boundedness of weighted Hardy operators presented in Theorem 13 is prepared by our estimations in Theorem 10. It is in fact a consequence of Theorem 10. We find it useful to divide this consequence into two parts. First, in Theorem 11, we reformulate Theorem 10 in the form to emphasize that we have pointwise estimates of Hardy operators $H_{w}^{\alpha(\cdot)}$ and $\mathscr{H}_{w}^{\alpha(\cdot)}$ in terms of the Morrey norm of the function $f$. Then as an immediate consequence of Theorem 11 we formulate Theorem 13 for global Morrey spaces.

Theorem 11. Let $p \in \mathscr{P}_{0, \infty}\left(\mathbb{R}^{n}\right)$ and $1 \leq p_{-} \leq p_{+}<\infty$. Let also the weight $w$ satisfy the conditions

$$
w \in \underline{W}\left(\mathbb{R}_{+}\right), \quad \frac{\varphi(0, \cdot)}{w(\cdot)} \in \underline{W}\left(\mathbb{R}_{+}\right),
$$

in the case of the operator $H_{w}^{\alpha(\cdot)}$, and the conditions

$$
\frac{1}{w} \in \bar{W}\left(\mathbb{R}_{+}\right), \quad \frac{\varphi(0, \cdot)}{w(\cdot)} \in \bar{W}\left(\mathbb{R}_{+}\right) .
$$

in the case of the operator $\mathscr{H}_{w}^{\alpha(\cdot)}$. The conditions

$$
\begin{aligned}
& \int_{0}^{\varepsilon} \frac{t^{\left(n / p^{\prime}(0)\right)-1} \varphi(0, t)}{w(t)} d t<\infty \\
& \int_{\varepsilon}^{\infty} \frac{t^{-(n / p(\infty))-1} \varphi(0, t)}{w(t)} d t<\infty
\end{aligned}
$$

with $\varepsilon>0$, are sufficient for the Hardy operators $H_{w}^{\alpha(\cdot)}$ and $\mathscr{H}_{w}^{\alpha(\cdot)}$, respectively, to be defined on the space $\mathscr{L}_{0 ; l o c}^{p(\cdot), \varphi(\cdot)}\left(\mathbb{R}^{n}\right)$. Under these conditions,

$$
\begin{aligned}
& \left|H_{w}^{\alpha(\cdot)} f(x)\right| \leq C K_{w, \alpha}(x)\|f\|_{\mathscr{L}_{0 ; l o c}^{p(\cdot),(\cdot)}}, \\
& \left|\mathscr{H}_{w}^{\alpha(\cdot)} f(x)\right| \leq C \mathscr{K}_{w, \alpha}(x)\|f\|_{\mathscr{L}_{0 ; l o c}^{p(\cdot), \varphi(\cdot)}},
\end{aligned}
$$

where

$$
\begin{aligned}
K_{w, \alpha}(x) & =|x|^{\alpha(x)-n} w(|x|) \mathscr{A}(|x|) \\
& =|x|^{\alpha(x)-n} w(|x|) \int_{0}^{|x|} \frac{t^{\left(n / p_{*}^{\prime}(t)\right)-1} \varphi(0, t)}{w(t)} d t, \\
\mathscr{K}_{w, \alpha}(x) & =|x|^{\alpha(x)} w(|x|) \int_{|x|}^{\infty} \frac{t^{-\left(n / p_{*}(t)\right)-1} \varphi(0, t)}{w(t)} d t .
\end{aligned}
$$

Proof. The sufficiency of the conditions in (51) and estimates in (52) follows from (36) under the choice $v(t)=w(t)$, for the operator $H_{w}^{\alpha(\cdot)}$ and $v(t)=1 / t^{n} w(t)$ for the operator $\mathscr{H}_{w}^{\alpha(\cdot)}$.

4.2. On the Necessity of the Conditions in (51). Observe that the conditions in (51) are natural in the sense that they are necessary under some additional assumptions on the function $\varphi$ defining the Morrey space.
Lemma 12. Let $p$ be as in Theorem 11 and $\varphi(0, \cdot) \in$ $\bar{W}\left(\left[0, \varepsilon_{0}\right]\right) \cap \underline{W}\left(\left[0, \varepsilon_{0}\right]\right)$ for some $\varepsilon_{0}>0$, and (30) holds. Then the conditions in (51) are necessary for the Hardy operators $H^{\alpha(\cdot)_{W}}$ and $\mathscr{H}^{\alpha(\cdot)_{W}}$, respectively, to be defined on the space $\mathscr{L}_{0 ; l o c}^{p(\cdot), \varphi(\cdot)}\left(\mathbb{R}^{n}\right)$.

Proof. Choose $f_{0}(x)=\left(\varphi(0,|x|) /|x|^{n / p(x)}\right) \chi_{B(0, R)}(x)$. Then $f \in \mathscr{L}_{0 ; \text { loc }}^{p(\cdot),(\cdot)}\left(\mathbb{R}^{n}\right)$ by Lemma 8 . It remains to note that the conditions in (51) are nothing else but the statement that $H_{w}^{\alpha(\cdot)} f_{0}$ and $\mathscr{H}_{w}^{\alpha(\cdot)} f_{0}$, respectively, exist.

4.3. Weighted $p \rightarrow q$ Norm Estimates for Hardy Operators. The statements of Theorem 13 are well known in the case of Lebesgue space, that is, in the case $\varphi \equiv 1$, with constant exponents, when $1<p<n / \alpha$; see for instance [27, pages 6, 54]. For the classical Morrey spaces $\mathscr{L}^{p, \lambda}\left(\mathbb{R}^{n}\right)$ with constant exponents $p$ and $\lambda$, statements of such type for Hardy operators have been obtained in $[17,19]$.

Hardy inequalities in the variable exponent Lebesgue spaces were studied in [34-36]; see also the references therein.

Note that, in contrast to variable exponent Lebesgue spaces, inequalities for the Hardy operators in Morrey spaces admit the case $\inf _{x} p(x)=1$ when $\varphi(0,0)=0$ in the case of local Morrey spaces and $\sup _{x} \varphi(x, 0)=0$ in the case of global Morrey spaces.

We suppose that the condition

$$
\sup _{r>0} \frac{[\min \{1, r\}]^{n / p(0)}}{\varphi(0, r)}<\infty
$$

holds, which ensures that the space $\mathscr{L}_{0, \mathrm{loc}}^{p(\cdot), \varphi(\cdot)}\left(\mathbb{R}^{n}\right)$ is nonempty by Lemma 6.

Theorem 13. Let $1 \leq p_{-} \leq p_{+}<\infty, 1 \leq q_{-} \leq q_{+}<\infty$ and $p \in \mathscr{P}_{0, \infty}(\Omega)$ as well as the functions $\varphi$ and $\psi$ satisfy the assumption (54). Let also the weight $w$ satisfy the conditions in (49) in the case of the operator $H_{w}^{\alpha(\cdot)}$ and the conditions in (50) in the case of the operator $\mathscr{H}_{w}^{\alpha(\cdot)}$. Then the operators $H_{w}^{\alpha(\cdot)}$ and $\mathscr{H}_{w}^{\alpha(\cdot)}$ are bounded from $\mathscr{L}_{0, \text { loc }}^{p(\cdot), \varphi(\cdot)}\left(\mathbb{R}^{n}\right)$ to $\mathscr{L}_{0, l o c}^{q(\cdot),(\cdot)}\left(\mathbb{R}^{n}\right)$, if

$$
K_{w, \alpha} \in \mathscr{L}_{l o c ; 0}^{q(\cdot),(\cdot)}\left(\mathbb{R}^{n}\right), \quad \mathscr{K}_{w, \alpha} \in \mathscr{L}_{l o c ; 0}^{q(\cdot), \psi(\cdot)}\left(\mathbb{R}^{n}\right),
$$

respectively. If $p$ and $\varphi(0, r)$ satisfy the assumptions of Corollary 9, then the conditions in (55) are also necessary for the boundedness of the operators $H_{w}^{\alpha(\cdot)}$ and $\mathscr{H}_{w}^{\alpha(\cdot)}$.

Proof. The sufficiency of the conditions in (55) for the boundedness follows from the estimates in (52).

As regards the necessity, the requirements in (55) are nothing else but the statement that

$$
\begin{aligned}
& H_{w}^{\alpha(\cdot)}\left(f_{0}\right) \in \mathscr{L}_{\mathrm{loc} ; 0}^{q(\cdot),(\cdot)}\left(\mathbb{R}^{n}\right), \\
& \mathscr{H}_{w}^{\alpha(\cdot)}\left(f_{0}\right) \in \mathscr{L}_{\mathrm{loc} ; 0}^{q(\cdot), \psi(\cdot)}\left(\mathbb{R}^{n}\right),
\end{aligned}
$$


respectively, where

$$
f_{0}(x)=\frac{\varphi(0,|x|)}{|x|^{n / p_{*}(|x|)}} .
$$

The function $f_{0}$ belongs to $\mathscr{L}_{0, \text { loc }}^{p(\cdot),(\cdot)}\left(\mathbb{R}^{n}\right)$ by Corollary 9 . Consequently, the conditions, (55) are necessary.

Corollary 14. Under the same assumptions on $p, \varphi$ and $w$ as in Theorem 13, the Hardy operators $H_{w}^{\alpha(\cdot)}$ and $\mathscr{H}_{w}^{\alpha(\cdot)}$ are bounded from the global Morrey space $\mathscr{L}^{p(\cdot), \varphi(\cdot)}\left(\mathbb{R}^{n}\right)$ to the global space $\mathscr{L}^{q(\cdot), \psi(\cdot)}\left(\mathbb{R}^{n}\right)$, if

$$
K_{w, \alpha} \in \mathscr{L}^{q, \psi}\left(\mathbb{R}^{n}\right), \quad \mathscr{K}_{w, \alpha} \in \mathscr{L}^{q, \psi}\left(\mathbb{R}^{n}\right),
$$

respectively.

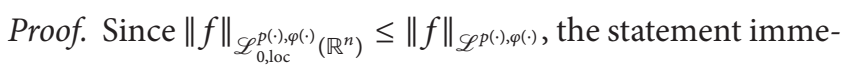
diately follows from the pointwise estimates in (52).

Remark 15. Theorem 18 is specifically a "Morrey-type" statement in the sense that the case of Lebesgue spaces (the case $\lambda=0$ ) is not included. This, in particular, is reflected in the admission of values $p(x)=1$ in Theorem 18, which is impossible for Lebesgue spaces.

4.4. Finding $\psi(0, r)$ by a Given $\varphi(0, r)$. The main theorem of the preceding section, Theorem 13 on the $\mathscr{L}_{0, \text { loc }}^{p(\cdot) \varphi(\cdot)}\left(\mathbb{R}^{n}\right) \rightarrow$ $\mathscr{L}_{0, \text { loc }}^{q(\cdot),(\cdot)}\left(\mathbb{R}^{n}\right)$ boundedness, provides a relation between the given function $\varphi(0, r)$ and $\psi(0, r)$ in an indirect form, via the conditions in (55). In the theorems below, under some additional assumptions on the functions $\varphi(0, r)$ we obtain the boundedness conditions in a form of Zygmund-type integral conditions imposed on $\varphi(0, r) / w(r)$ and give a direct relation between $\varphi(0, r)$ and $\psi(0, r)$.

In these theorems, we use the following assumptions on the function $\varphi(0, t)$ defining the data space $\mathscr{L}_{0, \text { loc }}^{p(\cdot),(\cdot)}\left(\mathbb{R}^{n}\right)$ :

$$
\varphi(0, r) \in \underline{W}\left(\mathbb{R}_{+}\right) \cap \mathbb{Z}^{\widetilde{\beta}_{0}, \widetilde{\beta}_{\infty}}\left(\mathbb{R}_{+}\right),
$$

where

$$
\begin{aligned}
& \tilde{\beta}_{0}=\frac{n}{p(0)}-\frac{n}{q(0)}-\alpha(0), \\
& \tilde{\beta}_{\infty}=\frac{n}{p(\infty)}-\frac{n}{q(\infty)}-\alpha(\infty)
\end{aligned}
$$

(see (A.18) for the definition of the classes $\mathbb{Z}^{\beta_{0}, \beta_{\infty}}\left(\mathbb{R}_{+}\right)$). Recall that the assumption $\varphi(0, r) \in \mathbb{Z}^{\widetilde{\beta}_{0}, \widetilde{\beta}_{\infty}}\left(\mathbb{R}_{+}\right)$in (59) may be equivalently rewritten in terms of the Matuszewska-Orlicz indices $m(\varphi), m_{\infty}(\varphi)$ of the function $\varphi(0, t)$ as

$$
m(\varphi)>\widetilde{\beta}_{0}, \quad m_{\infty}(\varphi)>\tilde{\beta}_{\infty}
$$

Theorem 16. Let $1 \leq p_{-} \leq p_{+}<\infty, 1 \leq q_{-} \leq q_{+}<\infty$, and $p, q \in \mathscr{P}_{0, \infty}\left(\mathbb{R}^{n}\right)$ as well as the function $\varphi$ satisfy the assumption in (54) and (59) and let the weight $w$ fulfill the conditions in (49). If

$$
\begin{array}{r}
\frac{\varphi(0, r)}{w(r)} \in \mathbb{Z}^{\beta_{0}, \beta_{\infty}}\left(\mathbb{R}_{+}\right) \quad \text { with } \beta_{0}=-\frac{n}{p^{\prime}(0)}, \\
\beta_{\infty}=-\frac{n}{p^{\prime}(\infty)},
\end{array}
$$

then the Hardy operator $H_{w}^{\alpha(\cdot)}$ is bounded from $\mathscr{L}_{0, l o c}^{p(\cdot), \varphi(\cdot)}\left(\mathbb{R}^{n}\right)$ to $\mathscr{L}_{0, l o c}^{q(\cdot),(\cdot)}\left(\mathbb{R}^{n}\right)$, with

$$
\psi(0, r)=r^{\alpha_{*}(r)-\left(n / p_{*}(r)\right)+\left(n / q_{*}(r)\right)} \varphi(0, r) .
$$

Proof. By (69), we have

$$
\begin{aligned}
& K_{w, \alpha}(x) \\
& \quad=|x|^{\alpha(x)-n} w(|x|) \int_{0}^{|x|} \frac{t^{\left(n / p_{*}^{\prime}(t)\right)-1} \varphi(0, t)}{w(t)} d t \\
& \quad \leq C|x|^{\alpha_{*}(|x|)-\left(n / p_{*}(|x|)\right)} \varphi(0,|x|) .
\end{aligned}
$$

To check that $K_{w, \alpha} \in \mathscr{L}_{\text {loc; } 0}^{q(\cdot) \varphi(\cdot)}\left(\mathbb{R}^{n}\right)$, by the definition in (20), we have to estimate the norm

$$
\left\||x|^{\alpha_{*}(|x|)-\left(n / p_{*}(|x|)\right)} \varphi(0,|x|) \chi_{B(0, r)}\right\|_{L^{q(\cdot)}} .
$$

We apply Lemma 2 with $a(x, r)=r^{\alpha_{*}(r)-\left(n / p_{*}(r)\right)} \varphi(0, r)$, which is possible by (59) and obtain

$$
\begin{aligned}
& \left\||x|^{\alpha_{*}(|x|)-\left(n / p_{*}(|x|)\right)} \varphi(0,|x|) \chi_{B(0, r)}\right\|_{L^{q(\cdot)}} \\
& \quad \leq C \int_{0}^{r} t^{\alpha_{*}(t)-\left(n / p_{*}(t)\right)+\left(n / q_{*}(t)\right)} \varphi(0, t) \frac{d t}{t} .
\end{aligned}
$$

Then by (59) we get

$$
\begin{gathered}
\left\||x|^{\alpha_{*}(|x|)-\left(n / p_{*}(|x|)\right)} \varphi(0,|x|) \chi_{B(0, r)}\right\|_{L^{q(\cdot)}} \\
\leq C r^{\alpha_{*}(r)-\left(n / p_{*}(r)\right)+\left(n / q_{*}(r)\right)} \varphi(0, r) .
\end{gathered}
$$

Therefore,

$$
\begin{gathered}
\left\||x|^{\alpha_{*}(|x|)-\left(n / p_{*}(|x|)\right)} \varphi(0,|x|)\right\|_{\mathscr{L}_{\mathrm{loc} ; 0}^{q(\cdot),(\cdot)}\left(\mathbb{R}^{n}\right)} \\
\leq C \frac{r^{\alpha_{*}(r)-\left(n / p_{*}(r)\right)+\left(n / q_{*}(r)\right)} \varphi(0, r)}{\psi(0, r)}
\end{gathered}
$$

and we arrive at (63).

Theorem 17. Let $1 \leq p_{-} \leq p_{+}<\infty, 1 \leq q_{-} \leq q_{+}<\infty$, $p, q \in \mathscr{P}_{0, \infty}\left(\mathbb{R}^{n}\right)$, and $\varphi$ satisfy (54) and (59) and let the weight $w$ fulfill (50). If

$$
\frac{\varphi(0, r)}{w(r)} \in \mathbb{Z}_{\gamma_{0}, \gamma_{\infty}}\left(\mathbb{R}_{+}\right) \quad \text { with } \gamma_{0}=\frac{n}{p(0)}, \gamma_{\infty}=\frac{n}{p(\infty)},
$$

then the Hardy operator $\mathscr{H}_{w}^{\alpha(\cdot)}$ is bounded from $\mathscr{L}_{0, l o c}^{p(\cdot), \varphi(\cdot)}\left(\mathbb{R}^{n}\right)$ to $\mathscr{L}_{0, \text { loc }}^{q(\cdot),(\cdot)}\left(\mathbb{R}^{n}\right)$, where $\psi(0, r)$ is the function (63). 
Proof. By (69), we have

$$
\begin{aligned}
& \mathscr{K}_{w, \alpha}(x)=|x|^{\alpha(x)} w(|x|) \int_{|x|}^{\infty} \frac{t^{-\left(n / p_{*}(t)\right)-1} \varphi(0, t)}{w(t)} d t \\
& \quad \leq C|x|^{\alpha_{*}(|x|)-\left(n / p_{*}(|x|)\right)} \varphi(0,|x|),
\end{aligned}
$$

after which the proof is the same as that of Theorem 16.

We single out an important case of non-weighted Hardy operators

$$
\begin{aligned}
& H^{\alpha(\cdot)} f(x)=|x|^{\alpha(x)-n} \int_{|y|<|x|} f(y) d y, \\
& \mathscr{H}^{\alpha(\cdot)} f(x)=|x|^{\alpha(x)} \int_{|y|>|x|} \frac{f(y) d y}{|y|^{n}}
\end{aligned}
$$

in variable exponent Morrey spaces of classical type, that is, with the function $\varphi(0, r)=\varphi_{\lambda / p}(0, r)$, defined by

$$
\varphi_{\lambda / p}(0, r)=\left\{\begin{array}{lll}
r^{\lambda / p(0)}, & r \leq 1 & =r^{\lambda / p_{*}(r)} \\
r^{\lambda / p(\infty)}, & r \leq 1 & =r^{\lambda / p_{*}(r)}
\end{array},\right.
$$

where $0<\lambda<n$ and then $\psi(0, r)=\varphi_{\lambda / q}(0, r)=r^{\lambda / q_{*}(r)}$ by (63).

Theorem 18. Let $\varphi_{\lambda / p}$ be of form (72), $1 \leq p(x) \leq p_{+}<\infty$, let $p, q, \alpha \in \mathscr{P}_{0, \infty}\left(\mathbb{R}^{n}\right)$ be such that $\alpha(0) \geq 0, \alpha(\infty) \geq 0$, and

$$
\frac{1}{q(0)}=\frac{1}{p(0)}-\frac{\alpha(0)}{n-\lambda}, \quad \frac{1}{q(\infty)}=\frac{1}{p(\infty)}-\frac{\alpha(\infty)}{n-\lambda} .
$$

Then the Hardy operators $H^{\alpha(\cdot)}$ and $\mathscr{H}^{\alpha(\cdot)}$ are bounded from $\mathscr{L}_{0, l o c}^{p(\cdot), \varphi_{\lambda / p}(\cdot)}\left(\mathbb{R}^{n}\right)$ to $\mathscr{L}_{0, l o c}^{q(\cdot), \varphi_{\lambda / q} i(\cdot)}\left(\mathbb{R}^{n}\right)$, if

$$
\alpha(0) p(0)<n-\lambda, \quad \alpha(\infty) p(\infty)<n-\lambda .
$$

In the case of $\inf _{x \in \mathbb{R}^{n}} p(x)=p(\infty)$, the conditions in (74) are also necessary.

Proof. In the sufficiency part, the theorem may be derived from Theorems 16 and 17, but we find it more convenient to derive it from more general statement of Theorem 13, since the functions $K_{w, \alpha}$ and $\mathscr{K}_{w, \alpha}$ may be explicitly calculated in this case and

$$
\begin{aligned}
& K_{w, \alpha}(x)=|x|^{\alpha(x)-\left((n-\lambda) / p_{*}(|x|)\right)}, \\
& \mathscr{K}_{w, \alpha}(x)=|x|^{\alpha(x)+\left(\lambda / p_{*}(|x|)\right)-\left(n / p_{*}^{\prime}(|x|)\right)} .
\end{aligned}
$$

Since $\alpha \in \mathscr{P}_{0, \infty}$, we have

$$
\begin{aligned}
& K_{w, \alpha}(x) \sim|x|^{\alpha_{*}(|x|)-\left((n-\lambda) / p_{*}(|x|)\right)}, \\
& \mathscr{K}_{w, \alpha}(x)=|x|^{\alpha_{*}(|x|)+\left(\lambda / p_{*}(|x|)\right)-\left(n / p_{*}^{\prime}(|x|)\right)},
\end{aligned}
$$

where the notation $\alpha_{*}(|x|)$ has the same meaning as in (10).
To check that $K_{w, \alpha} \in \mathscr{L}_{\text {loc;0 }}^{q(\cdot),(\cdot)}\left(\mathbb{R}^{n}\right)$, by the definition in (20) we have to estimate the norm $\||x|^{\alpha_{*}(|x|)-\left((n-\lambda) / p_{*}(|x|)\right)}$ $\chi_{B(0, r)}(x) \|_{q(\cdot)}$. To this end, we may apply Corollary 4 with $\nu(x)=\alpha_{*}(|x|)-\left((n-\lambda) / p_{*}(|x|)\right)$ and $p$ replaced by $q$. The assumptions on $\nu(x)$ of that corollary are satisfied if $0<\lambda<$ $n$ and $\max \{\alpha(0), \alpha(\infty)\}<n-\lambda$, which holds under the assumptions of the theorem. Thus, by Corollary 4 ,

$$
\begin{gathered}
\left\||x|^{\alpha_{*}(|x|)-\left((n-\lambda) / p_{*}(|x|)\right)} \chi_{B(0, r)}(x)\right\|_{q(\cdot)} \\
\leq C r^{\alpha_{*}(r)-\left((n-\lambda) / p_{*}(r)\right)+\left(n / q_{*}(r)\right)} .
\end{gathered}
$$

Then the required condition

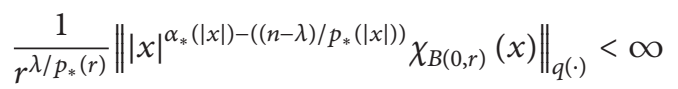

is guaranteed by (77) and (73).

Similarly the case of the operator $\mathscr{H}^{\alpha(\cdot)}$ is treated.

The necessity of the conditions (74) becomes evident if we note that in the case under consideration they are just the same as the conditions in (55) which are necessary by Theorem 13.

\section{Appendix}

\section{A. Zygmund-Bary-Stechkin (ZBS) Classes and Matuszewska-Orlicz (MO) Type Indices}

The reader can find more details and facts with proofs on the notions of this section for instance in [37-40]; see also the references therein. We recall some basic definitions and properties on which we based in our paper.

In the sequel, a non-negative function $f$ on $[0, \ell], 0<$ $\ell \leq \infty$, is called almost increasing (almost decreasing), if there exists a constant $C(\geq 1)$ such that $f(x) \leq C f(y)$ for all $x \leq y(x \geq y$, resp.). Equivalently, a function $f$ is almost increasing (almost decreasing), if it is equivalent to an increasing (decreasing, resp.) function $g$, that is, $c_{1} f(x) \leq$ $g(x) \leq c_{2} f(x), c_{1}>0, c_{2}>0$

Definition A.1. Let $0<\ell<\infty$.

(1) We denote by $W=W([0, \ell])$ the class of continuous and positive functions $\varphi$ on $(0, \ell]$ such that the limit $\lim _{x \rightarrow 0} \varphi(x)$ exists and is finite.

(2) We denote by $W_{0}=W_{0}([0, \ell])$ the class of almost increasing functions $\varphi \in W$ on $(0, \ell)$.

(3) We denote by $\bar{W}=\bar{W}([0, \ell])$ the class of functions $\varphi \in W$ such that $x^{a} \varphi(x) \in W_{0}$ for some $a=a(\varphi) \epsilon$ $\mathbb{R}^{1}$.

(4) We denote by $\underline{W}=\underline{W}([0, \ell])$ the class of functions $\varphi \in$ $W$ such that $\varphi(t) / t^{b}$ is almost decreasing for some $b \in$ $\mathbb{R}^{1}$.

Definition A.2. Let $0<\ell<\infty$.

(1) We denote by $W_{\infty}=W_{\infty}([\ell, \infty])$ the class of functions $\varphi$ which are continuous and positive and 
almost increasing on $[\ell, \infty)$ and which have the finite $\operatorname{limit}_{x \rightarrow \infty} \varphi(x)$.

(2) We denote by $\bar{W}_{\infty}=\bar{W}_{\infty}([\ell, \infty))$ the class of functions $\varphi \in W_{\infty}$ such $x^{a} \varphi(x) \in W_{\infty}$ for some $a=a(\varphi) \in \mathbb{R}^{1}$.

Finally, we denote by $\bar{W}\left(\mathbb{R}_{+}\right)$the set of functions on $\mathbb{R}_{+}$ whose restrictions onto $(0,1)$ are in $\bar{W}([0,1])$ and restrictions onto $[1, \infty)$ are in $\bar{W}_{\infty}([1, \infty))$. Similarly, the set $\underline{W}\left(\mathbb{R}_{+}\right)$is defined.

A.1. ZBS Classes and MO Indices of Weights at the Origin. In this subsection we assume that $\ell<\infty$.

Definition A.3. We say that a function $\varphi \in W_{0}$ belongs to the Zygmund class $\mathbb{Z}^{\beta}, \beta \in \mathbb{R}^{1}$, if

$$
\int_{0}^{x} \frac{\varphi(t)}{t^{1+\beta}} d t \leq c \frac{\varphi(x)}{x^{\beta}}, \quad x \in(0, \ell),
$$

and to the Zygmund class $\mathbb{Z}_{\gamma}, \gamma \in \mathbb{R}^{1}$, if

$$
\int_{x}^{\ell} \frac{\varphi(t)}{t^{1+\gamma}} d t \leq c \frac{\varphi(x)}{x^{\gamma}}, \quad x \in(0, \ell) .
$$

We also denote

$$
\Phi_{\gamma}^{\beta}:=\mathbb{Z}^{\beta} \bigcap \mathbb{Z}_{\gamma}
$$

the latter class being also known as Bary-Stechkin-Zygmund class [41].

It is known that the property of a function to be almost increasing or almost decreasing after the multiplication (division) by a power function is closely related to the notion of the so called Matuszewska-Orlicz indices. We refer for instance to to $[37,40,42-44]$, for the properties of the indices of such a type. For a function $\varphi \in \bar{W}$, the numbers

$$
\begin{aligned}
m(\varphi) & =\sup _{0<x<1} \frac{\ln \left(\limsup _{h \rightarrow 0}(\varphi(h x) / \varphi(h))\right)}{\ln x} \\
& =\lim _{x \rightarrow 0} \frac{\ln \left(\lim \sup _{h \rightarrow 0}(\varphi(h x) / \varphi(h))\right)}{\ln x} \\
M(\varphi) & =\sup _{x>1} \frac{\ln \left(\lim \sup _{h \rightarrow 0}(\varphi(h x) / \varphi(h))\right)}{\ln x} \\
& =\lim _{x \rightarrow \infty} \frac{\ln \left(\lim \sup _{h \rightarrow 0}(\varphi(h x) / \varphi(h))\right)}{\ln x}
\end{aligned}
$$

are known as the Matuszewska-Orlicz type lower and upper indices of the function $\varphi(r)$. Note that in this definition, $\varphi(x)$ needs not to be an $N$-function: only its behaviour at the origin is of importance. Observe that $0 \leq m(\varphi) \leq M(\varphi) \leq \infty$ for $\varphi \in W_{0}$, and $-\infty<m(\varphi) \leq M(\varphi) \leq \infty$ for $\varphi \in \bar{W}$, and the following formulas are valid:

$$
\begin{gathered}
m\left[x^{a} \varphi(x)\right]=a+m(\varphi), \\
M\left[x^{a} \varphi(x)\right]=a+M(\varphi), \quad a \in \mathbb{R}^{1}, \\
m\left([\varphi(x)]^{a}\right)=a m(\varphi), \\
M\left([\varphi(x)]^{a}\right)=a M(\varphi), \quad a \geq 0, \\
m\left(\frac{1}{\varphi}\right)=-M(\varphi), \quad M\left(\frac{1}{\varphi}\right)=-m(\varphi), \\
m(u v) \geq m(u)+m(v), \quad M(u v) \leq M(u)+M(v)
\end{gathered}
$$

for $\varphi, u, v \in \bar{W}$.

The following statement is known; see [37, Theorems 3.1, 3.2, and 3.5]. (In the formulation of [37, Theorem 5.4] it was supposed that $\beta \geq 0, \gamma>0$, and $\varphi \in W_{0}$. It is evidently true also for $\varphi \in \bar{W}$ and all $\beta, \gamma \in \mathbb{R}^{1}$, in view of formulas (A.5).)

Theorem A.4. Let $\varphi \in \bar{W}$ and $\beta, \gamma \in \mathbb{R}^{1}$. Then

$$
\varphi \in \mathbb{Z}^{\beta} \Longleftrightarrow m(\varphi)>\beta, \quad \varphi \in \mathbb{Z}_{\gamma} \Longleftrightarrow M(\varphi)<\gamma .
$$

Besides this

$$
\begin{aligned}
& m(\varphi)=\sup \left\{\mu>0: \frac{\varphi(x)}{x^{\mu}} \text { is almost increasing }\right\}, \\
& M(\varphi)=\inf \left\{\nu>0: \frac{\varphi(x)}{x^{\nu}} \text { is almost decreasing }\right\},
\end{aligned}
$$

and for $\varphi \in \Phi_{\gamma}^{\beta}$ the inequalities

$$
c_{1} x^{M(\varphi)+\varepsilon} \leq \varphi(x) \leq c_{2} x^{m(\varphi)-\varepsilon}
$$

hold with an arbitrarily small $\varepsilon>0$ and $c_{1}=c_{1}(\varepsilon), c_{2}=c_{2}(\varepsilon)$.

\section{A.2. ZBS Classes and MO Indices of Weights at Infinity}

Definition A.5. Let $-\infty<\alpha<\beta<\infty$. We put $\Psi_{\alpha}^{\beta}:=\widehat{\mathbb{Z}}^{\beta} \cap$ $\widehat{\mathbb{Z}}_{\alpha}$, where $\widehat{\mathbb{Z}}^{\beta}$ is the class of functions $\varphi \in \bar{W}_{\infty}$ satisfying the condition

$$
\int_{x}^{\infty}\left(\frac{x}{t}\right)^{\beta} \frac{\varphi(t) d t}{t} \leq c \varphi(x), \quad x \in(\ell, \infty),
$$

and $\widehat{\mathbb{Z}}_{\alpha}$ is the class of functions $\varphi \in W([\ell, \infty))$ satisfying the condition

$$
\int_{\ell}^{x}\left(\frac{x}{t}\right)^{\alpha} \frac{\varphi(t) d t}{t} \leq c \varphi(x), \quad x \in(\ell, \infty),
$$

where $c=c(\varphi)>0$ does not depend on $x \in[\ell, \infty)$. 
The indices $m_{\infty}(\varphi)$ and $M_{\infty}(\varphi)$ responsible for the behavior of functions $\varphi \in \Psi_{\alpha}^{\beta}([\ell, \infty))$ at infinity are introduced in the way similar to (A.4) as

$$
\begin{aligned}
& m_{\infty}(\varphi)=\sup _{x>1} \frac{\ln \left[\liminf _{h \rightarrow \infty}(\varphi(x h) / \varphi(h))\right]}{\ln x}, \\
& M_{\infty}(\varphi)=\inf _{x>1} \frac{\ln \left[\lim \sup _{h \rightarrow \infty}(\varphi(x h) / \varphi(h))\right]}{\ln x} .
\end{aligned}
$$

Properties of functions in the class $\Psi_{\alpha}^{\beta}([\ell, \infty))$ are easily derived from those of functions in $\Phi_{\beta}^{\alpha}([0, \ell])$ because of the following equivalence:

$$
\varphi \in \Psi_{\alpha}^{\beta}([\ell, \infty)) \Longleftrightarrow \varphi_{*} \in \Phi_{-\alpha}^{-\beta}\left(\left[0, \ell^{*}\right]\right),
$$

where $\varphi_{*}(t)=\varphi(1 / t)$ and $\ell_{*}=1 / \ell$. Direct calculation shows that

$$
\begin{aligned}
& m_{\infty}(\varphi)=-M\left(\varphi_{*}\right), \\
& M_{\infty}(\varphi)=-m\left(\varphi_{*}\right), \quad \varphi_{*}(t):=\varphi\left(\frac{1}{t}\right) .
\end{aligned}
$$

By (A.15) and (A.16), one can easily reformulate properties of functions of the class $\Phi_{\gamma}^{\beta}$ near the origin, given in Theorem A.4 for the case of the corresponding behavior at infinity of functions of the class $\Psi_{\alpha}^{\beta}$ and obtain that

$$
\begin{aligned}
& c_{1} t^{m_{\infty}(\varphi)-\varepsilon} \leq \varphi(t) \leq c_{2} t^{M_{\infty}(\varphi)+\varepsilon}, \quad t \geq \ell, \varphi \in \bar{W}_{\infty}, \\
& m_{\infty}(\varphi) \sup \left\{\mu \in \mathbb{R}^{1}: t^{-\mu} \varphi(t) \text { is almost increasing on }[\ell, \infty)\right\}, \\
& M_{\infty}(\varphi) \\
&=\inf \left\{\nu \in \mathbb{R}^{1}: t^{-v} \varphi(t) \text { is almost decreasing on }[\ell, \infty)\right\} .
\end{aligned}
$$

We say that a continuous function $\varphi$ in $(0, \infty)$ is in the class $\bar{W}_{0, \infty}\left(\mathbb{R}_{+}^{1}\right)$, if its restriction to $(0,1)$ belongs to $\bar{W}([0,1])$ and its restriction to $(1, \infty)$ belongs to $\bar{W}_{\infty}([1, \infty])$. For functions in $\bar{W}_{0, \infty}\left(\mathbb{R}_{+}^{1}\right)$, the notation

$$
\begin{aligned}
& \mathbb{Z}^{\beta_{0}, \beta_{\infty}}\left(\mathbb{R}_{+}^{1}\right)=\mathbb{Z}^{\beta_{0}}([0,1]) \cap \mathbb{Z}^{\beta_{\infty}}([1, \infty)), \\
& \mathbb{Z}_{\gamma_{0}, \gamma_{\infty}}\left(\mathbb{R}_{+}^{1}\right)=\mathbb{Z}_{\gamma_{0}}([0,1]) \cap \mathbb{Z}_{\gamma_{\infty}}([1, \infty))
\end{aligned}
$$

has an obvious meaning (note that in (A.18) we use $\mathbb{Z}^{\beta_{\infty}}([1, \infty))$ and $\mathbb{Z}_{\gamma_{\infty}}([1, \infty))$, not $\widehat{\mathbb{Z}}^{\beta_{\infty}}([1, \infty))$ and $\widehat{\mathbb{Z}}_{\gamma_{\infty}}$ $([1, \infty)))$. In the case where the indices coincide, that is, $\beta_{0}=\beta_{\infty}:=\beta$, we will simply write $\mathbb{Z}^{\beta}\left(\mathbb{R}_{+}^{1}\right)$ and similarly for $\mathbb{Z}_{\gamma}\left(\mathbb{R}_{+}^{1}\right)$. We also denote

$$
\Phi_{\gamma}^{\beta}\left(\mathbb{R}_{+}^{1}\right):=\mathbb{Z}^{\beta}\left(\mathbb{R}_{+}^{1}\right) \cap \mathbb{Z}_{\gamma}\left(\mathbb{R}_{+}^{1}\right) .
$$

Making use of Theorem A.4 for $\Phi_{\beta}^{\alpha}([0,1])$ and relations (A.16), one easily arrives at the following statement.
Lemma A.6. Let $\varphi \in \bar{W}\left(\mathbb{R}_{+}^{1}\right)$. Then

$$
\begin{aligned}
& \varphi \in \mathbb{Z}^{\beta_{0}, \beta_{\infty}}\left(\mathbb{R}_{+}^{1}\right) \Longleftrightarrow m(\varphi)>\beta_{0}, \quad m_{\infty}(\varphi)>\beta_{\infty}, \\
& \varphi \in \mathbb{Z}_{\gamma_{0}, \gamma_{\infty}}\left(\mathbb{R}_{+}^{1}\right) \Longleftrightarrow M(\varphi)<\gamma_{0}, \quad M_{\infty}(\varphi)<\gamma_{\infty} .
\end{aligned}
$$

\section{Acknowledgment}

The second author thanks Luleå University of Technology for financial support for research visits in February-April 2013.

\section{References}

[1] D. Cruz-Uribe and A. Fiorenza, Variable Lebesgue Spaces. Foundations and Harmonic Analysis, Birkhäauser, Boston, Mass, USA, 2013.

[2] L. Diening, P. Harjulehto, P. Hästö, and M. Růžička, Lebesgue and Sobolev Spaces with Variable Exponents, vol. 2017 of Lecture Notes in Mathematics, Springer, Berlin, Germany, 2011.

[3] M. Ružička, Electrorheological Fluids: Modeling and Mathematical Theory, vol. 1748 of Lecture Notes in Mathematics, Springer, New York, NY, USA, 2000.

[4] C. B. Morrey, "On the solutions of quasi-linear elliptic partial differential equations," American Mathematical Society, vol. 43, pp. 126-166, 1938.

[5] M. Giaquinta, Multiple Integrals in the Calculus of Variations and Nonlinear Elliptic Systems, Princeton University Press, Princeton, NJ, USA, 1983.

[6] A. Kufner, O. John, and S. Fučik, Function Spaces, Noordhoff International, Sussex, UK, 1977.

[7] M. E. Taylor, Tools for PDE: Pseudodifferential Operators, Paradifferential Operators, and Layer Potentials, vol. 81 of Mathematical Surveys and Monographs, AMS, Providence, RI, USA, 2000.

[8] H. Rafeiro, N. Samko, and S. Samko, "Morrey-campanato spaces: an overview," in Operator Theory, Pseudo-Differential Equations, and Mathematical Physics the Vladimir Rabinovich Anniversary Volume, vol. 228 of Operator Theory: Advances and Applications, Birkhäauser, Boston, Mass, USA, 2013.

[9] D. R. Adams and J. Xiao, "Nonlinear potential analysis on Morrey spaces and their capacities," Indiana University Mathematics Journal, vol. 53, no. 6, pp. 1631-1666, 2004.

[10] H. Arai and T. Mizuhara, "Morrey spaces on spaces of homogeneous type and estimates for $\square_{b}$ and the Cauchy-Szegö projection," Mathematische Nachrichten, vol. 185, pp. 5-20, 1997.

[11] V. I. Burenkov, H. V. Guliyev, and V. S. Guliyev, "Necessary and sufficient conditions for the boundedness of fractional maximal operators in local Morrey-type spaces," Journal of Computational and Applied Mathematics, vol. 208, no. 1, pp. 280-301, 2007.

[12] F. Chiarenza and M. Frasca, "Morrey spaces and HardyLittlewood maximal function," Rendiconti del Seminario Matematico, vol. 7, pp. 273-279, 1987.

[13] E. Nakai, "Hardy-Littlewood maximal operator, singular integral operators and the Riesz potentials on generalized Morrey spaces," Mathematische Nachrichten, vol. 166, pp. 95-103, 1994.

[14] D. K. Palagachev and L. G. Softova, "Singular integral operators, Morrey spaces and fine regularity of solutions to PDE's," Potential Analysis, vol. 20, no. 3, pp. 237-263, 2004. 
[15] J. Peetre, "On convolution operators leaving $\mathscr{L}^{p, \lambda}$ spaces invariant," Annali di Matematica Pura ed Applicata, vol. 72, no. 1, pp. 295-304, 1966.

[16] J. Peetre, "On the theory of $\mathscr{L}_{p, \lambda}$ spaces," Journal of Functional Analysis, vol. 4, no. 1, pp. 71-87, 1969.

[17] L.-E. Persson and N. Samko, "Weighted Hardy and potential operators in the generalized Morrey spaces," Journal of Mathematical Analysis and Applications, vol. 377, no. 2, pp. 792-806, 2011.

[18] N. Samko, "Weighted Hardy and singular operators in Morrey spaces," Journal of Mathematical Analysis and Applications, vol. 350, no. 1, pp. 56-72, 2009.

[19] N. Samko, "Weighted Hardy and potential operators in Morrey spaces," Journal of Function Spaces and Applications, vol. 2012, Article ID 678171, 21 pages, 2012.

[20] D. Lukkassen, L.-E. Persson, and S. Samko, "Weighted Hardy operators in complementary Morrey spaces," Journal of Function Spaces and Applications, vol. 20012, Article ID 283285, 19 pages, 2012.

[21] A. Almeida, J. Hasanov, and S. Samko, "Maximal and potential operators in variable exponent Morrey spaces," Georgian Mathematical Journal, vol. 15, no. 2, pp. 195-208, 2008.

[22] V. Kokilashvili and A. Meskhi, "Boundedness of maximal and singular operators in Morrey spaces with variable exponent," Armenian Journal of Mathematics, vol. 1, no. 1, pp. 18-28, 2008.

[23] V. Kokilashvili and A. Meskhi, "Maximal functions and potentials in variable exponent Morrey spaces with non-doubling measure," Complex Variables and Elliptic Equations, vol. 55, no. 8, pp. 923-936, 2010.

[24] V. S. Guliyev, J. J. Hasanov, and S. G. Samko, "Boundedness of the maximal, potential and singular operators in the generalized variable exponent Morrey spaces," Mathematica Scandinavica, vol. 107, no. 2, pp. 285-304, 2010.

[25] V. S. Guliyev, J. J. Hasanov, and S. G. Samko, "Boundedness of maximal, potential type, and singular integral operators in the generalized variable exponent Morrey type spaces $M^{p(\cdot), \theta(\cdot), w(\cdot)}(\Omega)$," Journal of Mathematical Sciences, vol. 170, no. 4, pp. 423-443, 2010.

[26] V. Guliev, J. Hasanov, and S. Samko, "Maximal, potential and singular operators in the local "complementary" variable exponent Morrey type spaces," Journal of Mathematical Analysis and Applications, vol. 401, no. 1, pp. 72-84, 2013.

[27] A. Kufner and L.-E. Persson, Weighted Inequalities of Hardy Type, World Scientific, River Edge, NJ, USA, 2003.

[28] V. Kokilashvili, A. Meskhi, and L.-E. Persson, Weighted Norm Inequalities for Integral Transforms with Product Kernels, Nova Science, New York, NY, USA, 2010.

[29] O. Kováč̌k and J. Rákosnı̌k, "On spaces $L^{p(x)}$ and $W^{k, p(x), "}$ Czechoslovak Mathematical Journal, vol. 41, no. 116, pp. 592-618, 1991.

[30] S. Samko, "On a progress in the theory of Lebesgue spaces with variable exponent: maximal and singular operators," Integral Transforms and Special Functions, vol. 16, no. 5-6, pp. 461-482, 2005.

[31] S. Samko, "Convolution type operators in $L^{p(x)}$," Integral Transforms and Special Functions, vol. 7, no. 1-2, pp. 123-144, 1998.

[32] A. Almeida and D. Drihem, "Maximal, potential and singular type operators on Herz spaces with variable exponents," Journal of Mathematical Analysis and Applications, vol. 394, no. 2, pp. 781-795, 2012.
[33] S. Samko, "Variable exponent Herz spaces," Mediterranean Journal of Mathematics, 2013.

[34] D. Cruz-Uribe and F. I. Mamedov, "On a general weighted Hardy type inequality in the variable exponent Lebesgue spaces," Revista Matematica Complutense, vol. 25, no. 2, pp. 335367, 2012.

[35] L. Diening and S. Samko, "Hardy inequality in variable exponent Lebesgue spaces," Fractional Calculus and Applied Analysis, vol. 10, no. 1, pp. 1-18, 2007.

[36] F. I. Mamedov and A. Harman, "On boundedness of weighted hardy operator in $L^{p(\cdot)}$ and regularity condition," Journal of Inequalities and Applications, vol. 2010, Article ID 837951, 14 pages, 2010.

[37] N. K. Karapetiants and N. G. Samko, "Weighted theorems on fractional integrals in the generalized Hölder spaces $H_{0}^{\omega}(\rho)$ via the indices $m_{\omega}$ and. $M_{\omega}$, Fractional Calculus and Applied Analysis, vol. 7, no. 4, pp. 437-458, 2004.

[38] L.-E. Persson, N. Samko, and P. Wall, "Quasi-monotone weight functions and their characteristics and applications," Journal of Inequalities and Applications, vol. 12, no. 3, pp. 685-705, 2012.

[39] N. G. Samko, S. G. Samko, and B. G. Vakulov, "Weighted Sobolev theorem in Lebesgue spaces with variable exponent," Journal of Mathematical Analysis and Applications, vol. 335, no. 1, pp. 560-583, 2007.

[40] N. Samko, "On non-equilibrated almost monotonic functions of the Zygmund-Bary-Stechkin class," Real Analysis Exchange, vol. 30, no. 2, pp. 727-745, 2005.

[41] N. K. Bari and S. B. Stechkin, "Best approximations and differential properties of two conjugate functions," Proceedings of the Moscow Mathematical Society, vol. 5, pp. 483-522, 1956 (Russian).

[42] S. G. Krein, I. Yu. Petunin, and E. M. Semenov, Interpolation of Linear Operators, Nauka, Moscow, Russia, 1978.

[43] W. Matuszewska and W. Orlicz, "On some classes of functions with regard to their orders of growth," Studia Mathematica, vol. 26, pp. 11-24, 1965.

[44] N. Samko, "Singular integral operators in weighted spaces with generalized Holder condition," Proceedengs of A.Razmadze Mathematical Institute, vol. 120, pp. 107-134, 1999. 


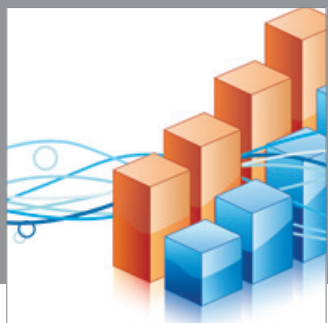

Advances in

Operations Research

mansans

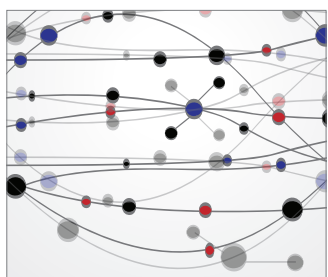

The Scientific World Journal
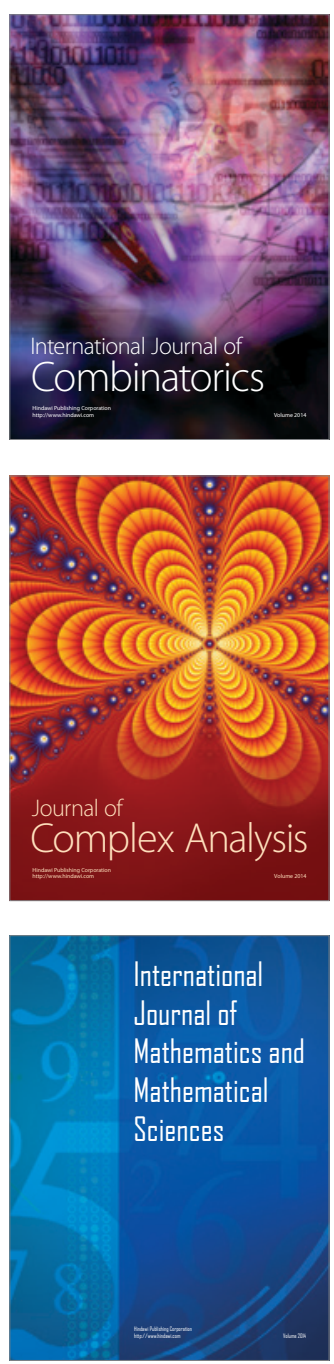
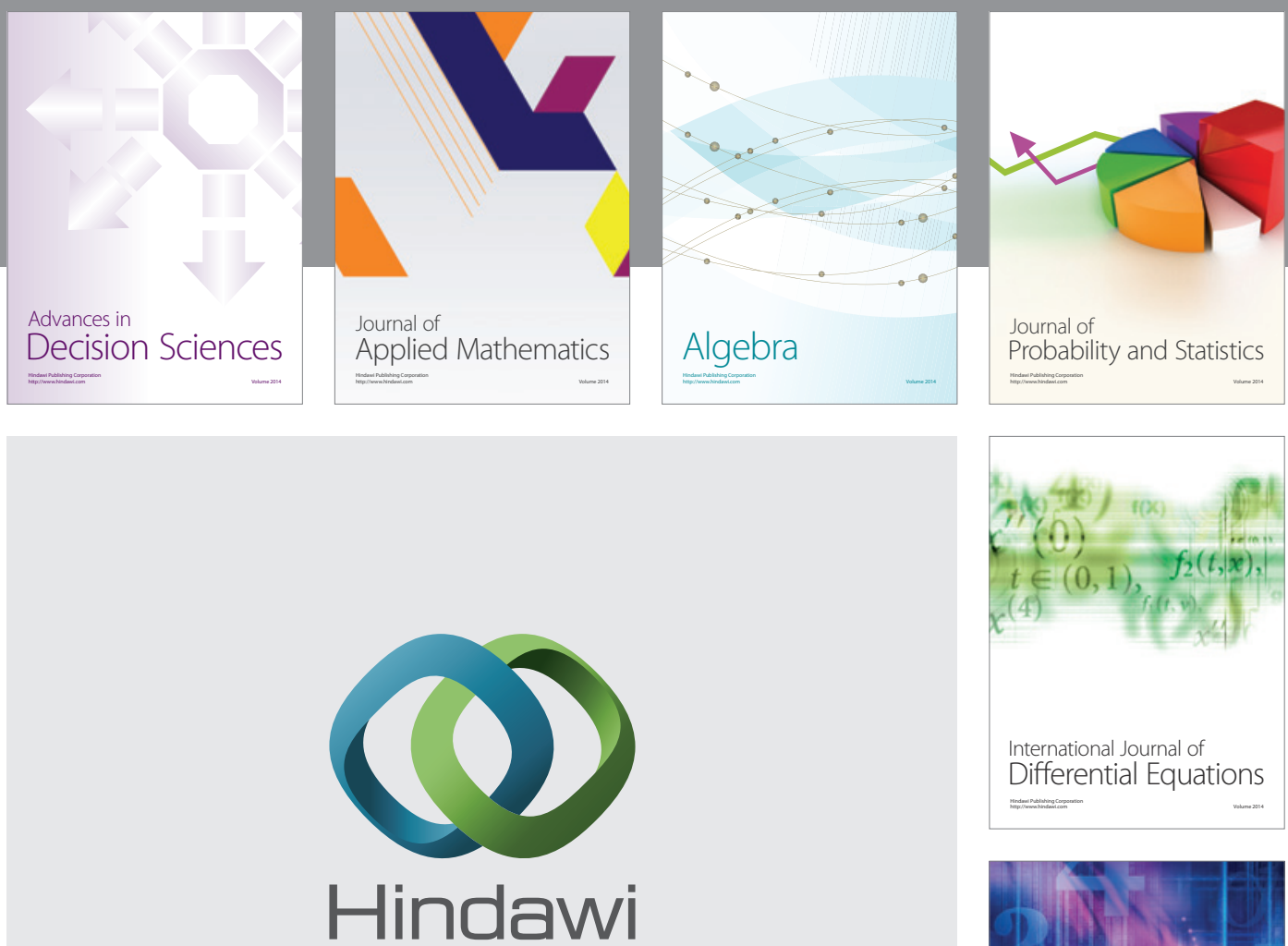

Submit your manuscripts at http://www.hindawi.com
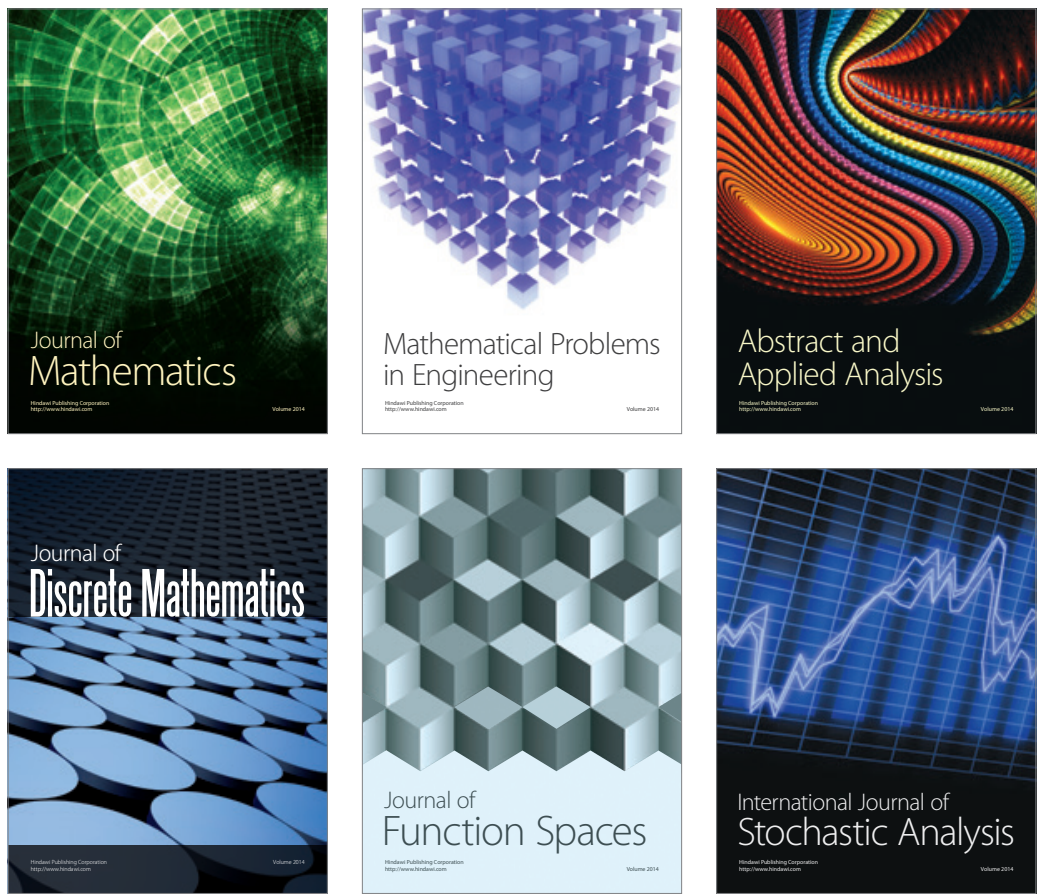

Journal of

Function Spaces

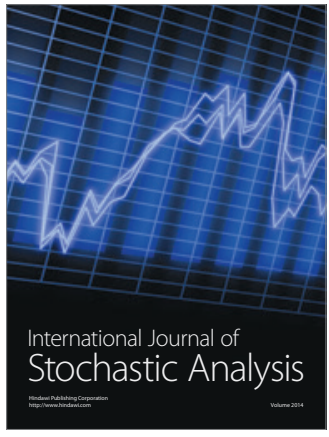

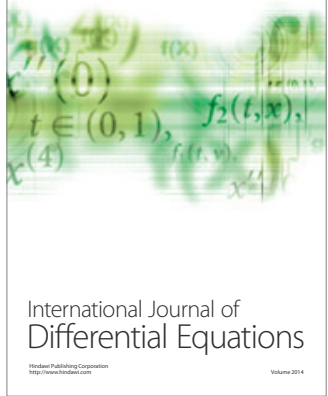
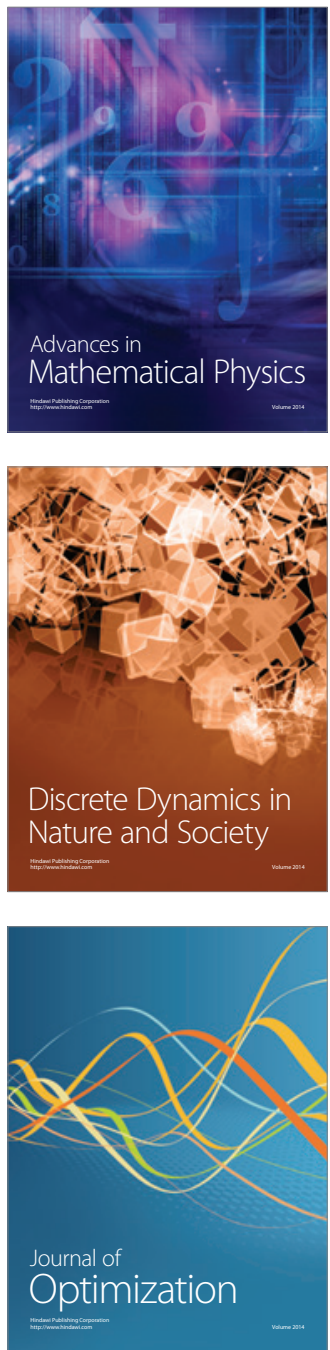